\title{
Micellization and adsorption of surfactant in a nonpolar liquid in micrometer scale geometries
}

\author{
F. Beunis, ${ }^{1, a)}$ F. Strubbe, ${ }^{1}$ M. Marescaux,${ }^{1}$ K. Neyts, ${ }^{1}$ and A. R. M. Verschueren ${ }^{2}$ \\ ${ }^{1}$ Department of Electronics and Information Systems, Ghent University, B-9000 Ghent, Belgium \\ ${ }^{2}$ Philips Research Laboratories, 5656 AE Eindhoven, The Netherlands
}

(Received 9 July 2010; accepted 28 September 2010; published online 3 November 2010)

\begin{abstract}
Mixtures of nonpolar liquid and surfactant are used increasingly in applications with microscopic dimensions. However, most methods to characterize them are performed on bulk solutions. We measure electrical transient currents in thin layers of nonpolar liquid with surfactant and derive several properties from these measurements. This paper reports the results for different liquid layer thicknesses and surfactant concentrations. We observe a dependence on the layer thickness of the inverse micelle concentration, which cannot be explained by bulk micellization alone. A model including surface adsorption is proposed that describes surfactant behavior in microscale geometries. (C) 2010 American Institute of Physics. [doi:10.1063/1.3503968]
\end{abstract}

Surfactants are added to nonpolar liquids as lubricants, dispersants, and charging agents in applications such as motor oils, ${ }^{1}$ inks, ${ }^{2}$ toners, ${ }^{3}$ developers, ${ }^{4}$ and ceramic processing. ${ }^{5}$ Surfactant molecules are adsorbed at the surfaces of colloidal particles ${ }^{6}$ and of the container. ${ }^{7}$ They also self-aggregate, forming inverse micelles (IMs) ${ }^{8}$ In most applications with macroscopic dimensions the bulk processes are independent from the adsorption at confining interfaces. Nonpolar liquids with surfactants are however used in an increasing number of applications with microscopic dimensions, such as electrophoretic displays ${ }^{9}$ or microfluidics, ${ }^{10}$ where bulk properties may be dependent on the geometry and the properties of the confining surface.

To characterize this dependence we perform transient current measurements ${ }^{11}$ on layers with different thicknesses of mixtures with different concentrations of surfactant in nonpolar liquid. These measurements allow to estimate the mobility and concentration of charged IMs. Using assumptions about IM formation and charging, we derive properties of the IMs, such as their effective radius and aggregation number, and of the solution, such as the concentration of IMs, the ratio between charged and neutral IMs and the critical micelle concentration $(\mathrm{cmc})$. We observe a dependence of the IM concentration on the thickness of the liquid layer. This dependency cannot be explained by micellization. We propose a model which combines bulk micellization and surface adsorption. This model is consistent with the measurements, and by fitting we estimate the surface density of adsorbed surfactant molecules.

The measured devices (Fig. 1) consist of two glass plates, coated with a transparent electrode of indium-tinoxide with surface area $S_{e l}=1 \mathrm{~cm}^{2}$. In a first series of devices, the plates are spincoated with a thin (around $50 \mathrm{~nm}$ ) layer of polyimide (PI) at the electrode side. In another series, the plates are left uncoated. Spacers keep the two plates separated at a distance $d$. The plates are glued together by a pattern which encloses a surface area $S_{t o t}$, which varies between 2 and $4 \mathrm{~cm}^{2}$. The space between the plates is filled with a mixture of high purity (99.9\%) n-dodecane (Aldrich) and different weight percentages of OLOA 1200, which contains 50\% mineral oil and 50\% of the surfactant polyisobutylene succinimide (Chevron). ${ }^{12}$ The total weight fraction of surfactant molecules in this mixture is then $c$ $=1 / 2(\mathrm{wt} \%$ OLOA $/ 100 \mathrm{wt} \%)$. In pure dodecane we expect few free charges, because of the low dielectric permittivity $(\varepsilon=2) .{ }^{13}$ The presence of surfactant facilitates the existence of univalent free charges in IMs. ${ }^{13,14}$ The devices are initially shortcircuited for a sufficient amount of time to obtain a homogeneous distribution of charges. At time $t=0$, a voltage step to $V_{A}=3 \mathrm{~V}$ is applied and the resulting current is measured.

This voltage is high enough to separate the charges, ${ }^{15}$ and allows to estimate the average concentration of charged IMs of one polarity $\bar{n}_{+}$from the integral of the measured transient current $I(t):^{15, \overline{16}}$

$$
\bar{n}_{ \pm}=\frac{1}{e S_{e l} d} \int_{0}^{\tau} I(t) d t
$$

where $e$ is the elementary charge and $\tau$ is the duration of the transient phase. We only integrate until time $\tau$ because there is a steady state current after the transient phase ${ }^{17}$ due to the generation of additional charged IMs (Ref. 17) or to electrode processes. ${ }^{18}$ The difference between the transient current, which changes quickly over time, and the steady state current, which is several orders of magnitude lower and almost constant, can easily be determined visually, especially for high voltages. Due to the low value of the steady state current, the effect of the choice of $\tau$ and the error due to the generation current during the transient phase can be neglected. From the initial value of the transient current, we

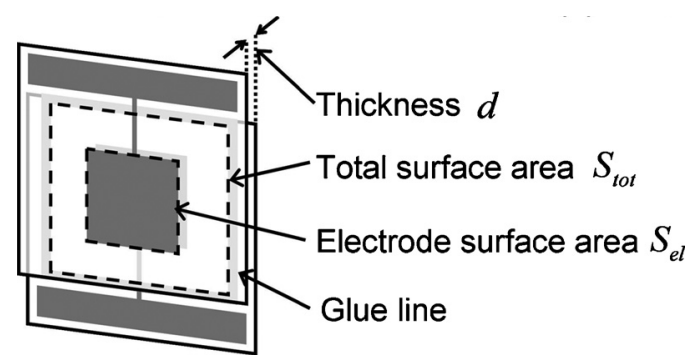

FIG. 1. Schematic drawing of the devices used in the measurements. 


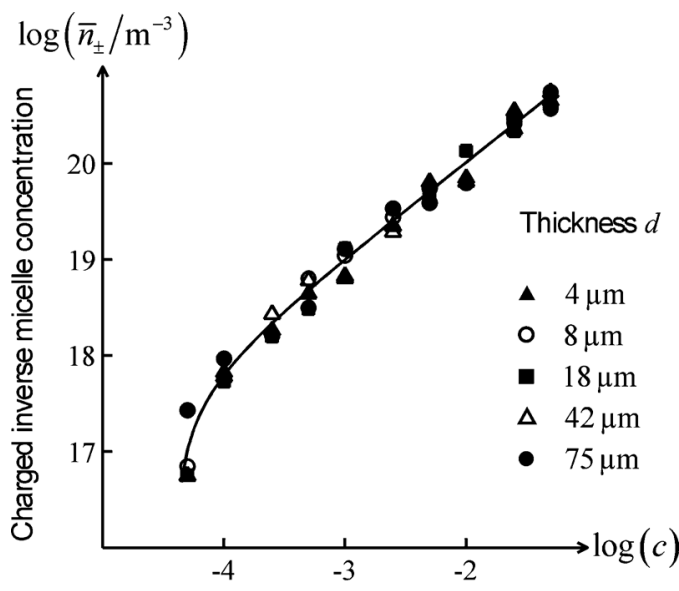

Surfactant weight fraction

FIG. 2. Measured (symbols) and fitted (line) concentrations of charged IMs, in devices with a PI coating, for different thicknesses.

can estimate the mobility $\mu$ of the charged IMs: ${ }^{15,16}$

$$
\mu=\frac{I(t=0) d}{2 e \bar{n}_{ \pm} V_{A} S_{e l}} .
$$

The concentration of charges in devices with PI is approximately proportional to the surfactant weight fraction (Fig. 2). The same observation can be made for devices without PI at the higher surfactant concentrations, but for lower concentrations there is a dependence on the thickness of the device (Fig. 3). The mobility is approximately the same for all measurements, averaging $8.8 \times 10^{-10} \mathrm{~m}^{2} \mathrm{~V}^{-1} \mathrm{~s}^{-1}$.

The experimental observations allow the estimation of a number of properties of the IMs. We assume that the shape of all IMs is spherical, and can be described by the same effective radius $R$. The mobility of the charged IMs is then related to their valency $Z$, their effective radius $R$ and to the viscosity of the liquid $\eta: \mu=(Z e) /(6 \pi \eta R)$. Knowing the viscosity of dodecane $\left(\eta=1.4 \times 10^{-3} \mathrm{~kg} \mathrm{~m}^{-1} \mathrm{~s}^{-1}\right)$, and the fact that the IMs are univalent, ${ }^{19,20}$ the effective radius of the IMs is $R=(Z e) /(6 \pi \eta \mu)=7.6 \mathrm{~nm}$. This value agrees with the size of an OLOA $1200 \mathrm{IM}$ in dodecane, reported in other works. ${ }^{19,21}$ Using this radius, the average volume of an IM is

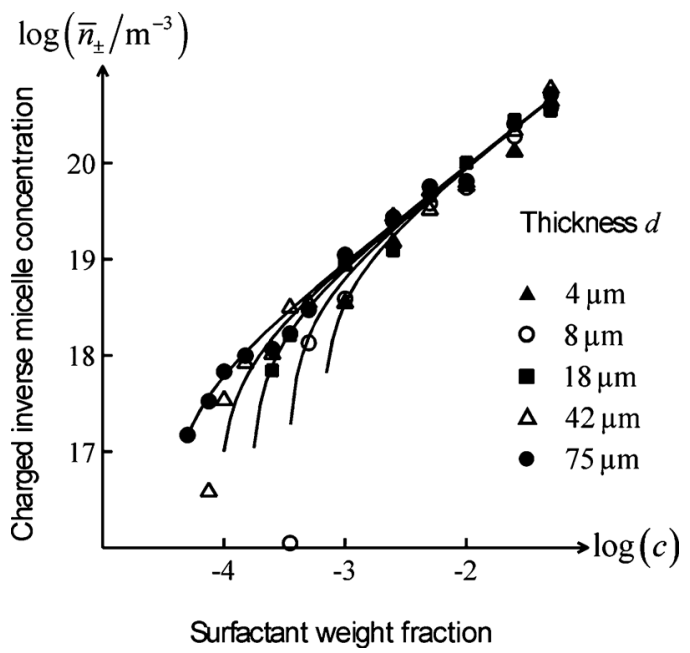

FIG. 3. Measured (symbols) and fitted (line) concentrations of charged IMs, in devices without a PI coating, for different thicknesses.
$1840 \mathrm{~nm}^{3}$. Assuming that an IM has the same density as pure dodecane $\left(\rho_{m}=750 \mathrm{~kg} \mathrm{~m}^{-3}\right)$, its mass $m_{\text {mic }}$ can be estimated as $m_{m i c}=(4 / 3) \pi \rho_{m} R^{3}=1.38 \times 10^{-21} \mathrm{~kg}$. The mass of one surfactant molecule $m_{m o l}$ is $2.8 \times 10^{-24} \mathrm{~kg}$. The aggregation number $N$, which is the average number of surfactant molecules per IM, is then $N=m_{\text {mic }} / m_{\text {mol }}=490$.

If all IMs consist of the same number of surfactant molecules, one expects the total concentration $\bar{n}_{t o t}$ of both charged and neutral IMs to increase proportionally with the surfactant weight fraction $c$ :

$$
\Delta \bar{n}_{t o t}=\frac{\rho_{m}}{m_{m o l} N} \Delta c .
$$

In devices with PI, the concentration of charged IMs increases proportionally with the surfactant weight fraction and therefore proportionally with the total IM concentration (Fig. 2). This indicates that the charged IMs are the result of a disproportionation reaction. ${ }^{17,22}$ If the equilibrium constant of the reaction is $K=\bar{n}_{ \pm}^{2} / \bar{n}_{0}^{2}$, the relation between the concentration of charged IMs and the total IM concentration is

$$
\bar{n}_{ \pm}=\frac{\sqrt{K}}{1+2 \sqrt{K}} \bar{n}_{t o t} .
$$

Figure 2 shows that under a certain surfactant weight fraction the charged IM concentration is zero. We explain this by assuming that a minimal weight fraction (the $\mathrm{cmc}$ ) of surfactant molecules $c_{\mathrm{cmc}}$ is necessary before IMs can be formed. Using this assumption and Eq. (3) results in

$$
\bar{n}_{\text {tot }}=\frac{\rho_{m}}{m_{m o l} N}\left(c-c_{\mathrm{cmc}}\right) .
$$

Combining Eqs. (4) and (5), the charged IM concentration can be expressed in function of the surfactant weight fraction as follows:

$$
\bar{n}_{ \pm}=\frac{\sqrt{K}}{1+2 \sqrt{K}} \frac{\rho_{m}}{m_{m o l} N}\left(c-c_{\mathrm{cmc}}\right) .
$$

The unknown equilibrium constant $K$ and the $\mathrm{cmc} c_{\mathrm{cmc}}$ can be used to fit Eq. (6) with the measurements of devices with a PI coating. The best fit is plotted on Fig. 2 and is found for $K=3.9 \times 10^{-4}$ (corresponding to one positively and one negatively charged micelle in every 53 micelles) and $c_{\mathrm{cmc}}=4.1$ $\times 10^{-5}$ (expressed as a weight fraction of surfactant in dodecane). The obtained fraction of charged IMs (about $4 \%$ ) is lower than the upper limit of $2 \exp \left(-\lambda_{\mathrm{B}} / R\right) \approx 5 \%$, calculated by comparing the measured micelle radius of $7.6 \mathrm{~nm}$, separating the charge in the micelle core from charges in solution, with the Bjerrum distance $\lambda_{\mathrm{B}}$ in dodecane $(28 \mathrm{~nm})$.

Figure 3 indicates that, in the devices with uncoated electrodes, the concentration of charged IMs [and according to Eq. (4) also the total concentration of IMs] depends on the thickness of the device, which cannot be explained by bulk micellization. For each thickness there is a different critical value of the weight fraction of surfactant molecules below which no charge could be measured. In the devices with uncoated electrodes, we attribute the missing micelles in the bulk at low concentrations to two effects: a minimal fraction of surfactant molecules $c_{\mathrm{cmc}}$ in the bulk is needed before IMs can be formed and the surfactant preferably covers the surfaces of the structure up to a surface concentration of surfactant molecules $\nu$. The total mass of surfactant molecules is 
then distributed over three categories: IMs in the bulk, adsorbed at the two surfaces or individual surfactant molecules in the bulk

$$
c \rho_{m} S_{t o t} d=\bar{n}_{t o t} N m_{m o l} S_{t o t} d+2 \nu m_{m o l} S+c_{\mathrm{cmc}} \rho_{m} S_{t o t} d,
$$

where $S$ is the surface area (on one plate) on which molecules become adsorbed. The molecules may be adsorbed only at the electrodes $\left(S=S_{e l}\right)$, only at the glass $\left(S=S_{t o t}\right.$ $\left.-S_{e l}\right)$, or at the complete surface $\left(S=S_{t o t}\right)$. Combining Eq. (4) with Eq. (7), the concentration of charged micelles can be found as follows:

$$
\bar{n}_{ \pm}=\frac{\sqrt{K}}{1+2 \sqrt{K}} \frac{1}{N}\left[\frac{\rho_{m}}{m_{m o l}}\left(c-c_{\mathrm{cmc}}\right)-2 \nu \frac{S}{S_{t o t}} \frac{1}{d}\right] .
$$

The quantities $S, K, c_{\mathrm{cmc}}$, and $\nu$ can be used to fit Eq. (8) to the measurements. We do not see a dependence on $S_{t o t}$, which indicates that the surfactant is adsorbed both at the electrodes and at the glass, so that $S=S_{t o t}$, and $S_{t o t}$ disappears from Eq. (8). Fitting the slope of the curves in Fig. 3 yields $K=3.2 \times 10^{-4}$ (corresponding to one positively and one negatively charged micelle in every 58 micelles). This result agrees with the result for coated electrodes, which indicates that the charging process is independent of the surfaces. Fitting Eq. (8) to the measurements for low concentrations yields $\nu=0.3 \mathrm{~nm}^{-2}$. Finally, fitting the measurements at high thicknesses and low concentrations yields $c_{\mathrm{cmc}}=3.5 \times 10^{-5}$. The fact that an adsorbed surface layer is formed on uncoated electrodes and not on electrodes coated with a PI layer can be understood by considering the polarity of the surfaces. PI is a dielectric material, with a relative dielectric constant of only 3 , which is lower than the dielectric constant of 5.5 for uncoated glass. The difference with the relative dielectric constant of dodecane $(\varepsilon=2)$, and therefore the driving force for surface adsorption is small. The free charges in the electrodes make it much more likely for the surfactant molecules to adsorb on this surface.

This work shows that values obtained from characterization of a bulk solution of surfactant in nonpolar liquid should be used carefully when applied to geometries with microme- ter scale dimensions. When the ratio between volume and surface area is small, bulk and surface effects cannot be assumed to be independent of each other. We observe that the IM concentrations derived from transient current measurements are dependent on the weight fraction of surfactant molecules and on the thickness of the device. We explain the dependence on the surfactant concentration as micellization, and the dependence on the layer thickness as a consequence of surface adsorption of surfactant molecules. This model can be used to estimate several properties of the micellar solution and of the adsorbed surface layer.

${ }^{1}$ I. F. Blagovidov, V. P. Lapin, G. L. Trofimpva, and G. I. Shor, Chem. Technol. Fuels Oils 7, 439 (1971).

${ }^{2}$ H. J. Spinelli, Adv. Mater. 10, 1215 (1998).

${ }^{3}$ I. Chen, J. Mort, and M. A. Machonkin, Langmuir 13, 5036 (1997).

${ }^{4}$ K. A. Pearlstine, J. Imaging Sci. 35, 326 (1991).

${ }^{5}$ V. M. B. Moloney, D. Parris, and M. J. Edirisinghe, J. Am. Ceram. Soc. 78, 3225 (1995).

${ }^{6}$ B. L. Papke and L. M. Robinson, Langmuir 10, 1741 (1994).

${ }^{7}$ B. Y. Zhu and T. R. Gu, Adv. Colloid Interface Sci. 37, 1 (1991).

${ }^{8}$ S. P. Moulik, Curr. Sci. 71, 368 (1996).

${ }^{9}$ B. Comiskey, J. D. Albert, H. Yoshizawa, and J. Jacobson, Nature (London) 394, 253 (1998).

${ }^{10}$ J. S. H. Lee, I. Barbulovic-Nad, Z. Wu, X. Xuan, and D. Li, J. Appl. Phys. 99, 054905 (2006).

${ }^{11}$ V. Novotny, J. Electrochem. Soc. 133, 1629 (1986).

${ }^{12}$ R. E. Kornbrekke, I. D. Morrison, and T. Oja, Langmuir 8, 1211 (1992).

${ }^{13}$ I. D. Morrison, Colloids Surf., A 71, 1 (1993).

${ }^{14}$ A. S. Dukhin and P. J. Goetz, J. Electroanal. Chem. 588, 44 (2006).

${ }^{15}$ F. Beunis, F. Strubbe, K. Neyts, and A. R. M. Verschueren, Appl. Phys. Lett. 90, 182103 (2007).

${ }^{16}$ F. Beunis, F. Strubbe, M. Marescaux, K. Neyts, and A. R. M. Verschueren, Appl. Phys. Lett. 91, 182911 (2007).

${ }^{17}$ F. Strubbe, A. R. M. Verschueren, L. J. M. Schlangen, F. Beunis, and K. Neyts, J. Colloid Interface Sci. 300, 396 (2006).

${ }^{18}$ S. Jayaram and J. D. Cross, J. Electrost. 29, 55 (1992).

${ }^{19}$ A. R. M. Verschueren, P. H. L. Notten, L. J. M. Schlangen, F. Strubbe, F Beunis, and K. Neyts, J. Phys. Chem. B 112, 13038 (2008).

${ }^{20}$ F. Beunis, F. Strubbe, M. Marescaux, J. Beeckman, K. Neyts, and A. R. M. Verschueren, Phys. Rev. E 78, 011502 (2008).

${ }^{21}$ J. Kim, J. L. Anderson, S. Garoff, and L. J. M. Schlangen, Langmuir 21, 8620 (2005).

${ }^{22}$ M. F. Hsu, E. R. Dufresne, and D. A. Weitz, Langmuir 21, 4881 (2005). 